\title{
Prevalência de sintomas depressivos e fatores associados em idosos no norte de Minas Gerais: um estudo de base populacional
}

Prevalence of depressive symptoms and associated factors among elderly in northern Minas Gerais: a population-based study

Gizele Carmem Fagundes Ramos', Jair Almeida Carneiro', Ana Teresa Fernandes Barbosa', José Marcio Girardi Mendonça', Antônio Prates Caldeira'

\section{RESUMO}

Objetivo: Avaliar a prevalência e os fatores associados aos sintomas depressivos em idosos não institucionalizados. Métodos: Estudo transversal, analítico, de base populacional, cujos dados foram coletados entre maio e julho de 2013, em visitas domiciliares. Foi aplicado um questionário com variáveis sociodemográficas, comorbidades, utilização de serviços de saúde, escala de fragilidade (Edmonton Frail Scale), teste Timed Get Up and Go e a Escala de Depressão Geriátrica (Geriatric Depression Scale - GDS-15). Para análise estatística, as variáveis foram dicotomizadas. Conduziram-se análises bivariadas (teste qui-quadrado de Pearson) adotando-se nível de significância menor que 0,20 para inclusão das variáveis independentes no modelo múltiplo. O modelo final foi gerado por meio de análise de regressão logística múltipla e as variáveis mantidas apresentaram associação com sintomas depressivos em um nível de significância de 0,05 ( $p<0,05)$. Resultados: A prevalência de sintomas depressivos foi de 27,5\%. As variáveis independentes associadas a sintomas depressivos foram: não ter companheiro (a) $(\mathrm{OR}=1,81$; IC 95\% 1,214-2,713), não saber ler ( $\mathrm{OR}=1,84$; IC 95\% 1,19-2,836), percepção negativa sobre a própria saúde (OR = 2,12; IC 95\% 1,373-3,256), tabagismo ( $O R=2,31$; IC 95\% 1,208-4,431), alto risco de quedas $(\mathrm{OR}=1,78 ; \mathrm{IC} 95 \%$ 1,000-3,184) e fragilidade (OR $=2,38 ; \mathrm{IC} 95 \%$ 1,510-3,754).

\section{Palavras-chave}

Idoso, depressão, prevalência, epidemiologia.
Conclusões: A alta prevalência de sintomas depressivos identificada entre idosos comunitários alerta para a necessidade de maiores cuidados com a população idosa.

\section{ABSTRACT}

Objective: To evaluate the prevalence and the factors associated with depressive symptoms in the noninstitutionalized elderly. Methods: A cross sectional analytical study populationbased, conducted between May and July 2013, through a household survey. A questionnaire with sociodemographic variables, comorbidities, use of health services, scale fragility (Edmonton Frail Scale), Timed Get Up and Go test and Geriatric Depression Scale GDS-15 was applied. For statistical analysis, the variables were dichotomized. Bivariate analyzes (chi-square test) were conducted adopting a significance level lower than 0.20 for inclusion of independent variables in the multiple model. The final model was generated using multivariate logistic re-

1 Universidade Estadual de Montes Claros (Unimontes), Programa de Pós-Graduação Stricto Sensu em Ciências da Saúde.

Endereço para correspondência: Gizele Carmem Fagundes Ramos

Hospital Universitário Clemente de Faria, Programa de Pós-Graduação em Ciências da Saúde (PPGCS)

Av. Cula Mangabeira, 562, Santo Expedito

39401-001 - Montes Claros, MG, Brasil

E-mail: gizelecarmem@yahoo.com.br 


\section{Keywords}

Senior, depression, prevalence, epidemiology. gression and the variables were associated with depressive maintained at a significance level of 0.05 ( $p<0.05$ ) symptoms. Results: The prevalence of depressive symptoms was $27.5 \%$. Independent variables associated with depressive symptoms were: not having a partner (OR $=1.81,95 \% \mathrm{Cl} 1.214$ to 2.713$)$, not reading $(\mathrm{OR}=1.84,95 \% \mathrm{Cl} 1.19$ to 2.836$)$, having negative perception about their own health ( $\mathrm{OR}=2.12,95 \% \mathrm{Cl} 1.373$ to 3.256$)$, smoking $(\mathrm{OR}=2.31,95 \%$ $\mathrm{Cl} 1.208$ to 4.431 ), high risk of falls ( $\mathrm{OR}=1.78,95 \% \mathrm{Cl} 1.000$ to 3.184 ) and frailty $(\mathrm{OR}=2.38,95 \%$ $\mathrm{Cl} 1.510$ to 3.754 ). Conclusions: The high prevalence of depressive symptoms among community-dwelling elders identified alert to the need for better care of the elderly population.

\section{INTRODUÇÃO}

A depressão na terceira idade é um problema de saúde mental bastante comum, com profundo impacto negativo sob todos os aspectos na vida do idoso, bem como em sua família e na comunidade ${ }^{1,2}$. Tal impacto está associado aos piores desfechos em saúde, ao comprometimento da saúde física, à maior utilização de serviços de saúde e a maiores custos, além do aumento do risco de morte ${ }^{3}$.

A depressão maior é caracterizada por humor deprimido, perda do interesse ou prazer, alterações do funcionamento biológico, com repercussões importantes na vida do indivíduo e com duração, sem tratamento, de meses a anos. Para definição de seu diagnóstico, utilizam-se os critérios da Classificação Internacional de Doenças (CID-10) e/ou do Diagnostic and Satistical Manual of Mental Disorders (DSM)'.

É importante destacar que o humor deprimido ou tristeza não são essenciais para o diagnóstico das síndromes depressivas nos idosos. Muitas vezes eles têm dificuldade de verbalizar uma tristeza e podem referir irritabilidade, falta de sentimentos e emoções, culpa, desamparo, perda do interesse ou prazer em atividades que eram antes consideradas prazerosas ${ }^{4}$.

O termo "depressão subsindrômica" se aplica a indivíduos com sintomas depressivos, mas que não preenchem critérios para depressão maior, podendo englobar distimia e depressão menor ${ }^{5}$. A expressão "sintomas depressivos clinicamente significativos" (SDCS) refere-se a uma ampla categoria de indivíduos com sintomas depressivos detectados por meio de escalas de triagem, podendo incluir aqueles com depressão maior, depressão menor ou distimia6. A maioria dos idosos com SDCS não preenche os critérios diagnósticos de depressão maior, mas a morbidade cumulativa associada com depressão menor na população excede aquela para depressão maior?.

Estudos demonstram associação entre sintomatologia depressiva em idosos e fatores sociodemográficos como: sexo feminino ${ }^{8-11}$, idade avançada $a^{8,9,12}$, baixa escolaridade ${ }^{8,10,12}$ e não ter companheiro conjugal $\left.\right|^{8}$. No que diz respeito às condições de saúde, são fatores descritos como associados aos sintomas depressivos: incapacidade funciona| ${ }^{8,12-18}$, percepção negativa da saúde ${ }^{8,12,17,18}$, tabagismo ${ }^{14,19}$, maior utilização de medicamentos ${ }^{16}$, insônia ${ }^{8,12}$ e comorbidades somáticas crônicas ${ }^{9,17,20}$. Apesar desses aspectos já apontados pela literatura, ainda existem lacunas, pois existem poucos estudos com abordagem de base populacional em regiões carentes e fora dos grandes centros metropolitanos.

No Brasil, entre idosos comunitários, a prevalência de depressão maior varia de 3\% a 15\% nas diversas regiões, enquanto a prevalência de sintomas depressivos varia de 13\% a 39\%, o que indica maior prevalência de sintomas depressivos em comparação com taxas relatadas na literatura internacional ${ }^{9}$, cuja média situa-se em torno de $13,5 \%{ }^{11}$. As discrepâncias entre os valores observados também ressaltam a necessidade de estudos regionais e de base populacional, para que as variáveis identificadas possam ser abordadas de maneira mais efetiva e imediata pelos gestores de saúde.

A alta prevalência de sintomas depressivos e depressão subsindrômica em idosos reforça ainda a importância de se investigá-los, por estarem associados a maior risco cardiovascular e outras doenças médicas, bem como com o risco de evolução para depressão maior?

Para a região norte do estado de Minas Gerais, são escassos os registros de avaliações sistemáticas ou avaliações de base populacional abordando sintomatologia depressiva e fatores associados, o que destaca o caráter original do estudo. Considerando a particularidade da região, que geograficamente está inserida na Região Sudeste do país, mas agrega municípios com baixos Índices de Desenvolvimento Humano (IDH) e possui características muito mais próximas da Região Nordeste, estudos locais, que avaliem as condições de vida e saúde da população idosa, têm o potencial de gerar novos conhecimentos e subsidiar a tomada de decisão para os gestores de saúde. Sendo assim, este trabalho objetivou avaliar a prevalência e os fatores associados aos sintomas depressivos em idosos comunitários em uma cidade do norte de Minas Gerais.

\section{MÉTODOS}

Este estudo é parte de uma análise das condições de saúde de idosos não institucionalizados, residentes no norte de Minas Gerais e foi conduzido no município de Montes Claros, principal polo urbano da região. Trata-se de estudo transver- 
sal, de base populacional, cujos dados foram coletados entre maio e julho de 2013, em visitas domiciliares.

O processo de amostragem foi probabilístico, por conglomerados e em dois estágios. No primeiro estágio, utilizou-se como unidade amostral o setor censitário e foram selecionados aleatoriamente 42 setores censitários, entre os 362 setores urbanos do município ${ }^{21}$. No segundo estágio, definiu-se o número de domicílios segundo a densidade populacional de indivíduos com idade $\geq 60$ anos. Nessa etapa, os setores com maior número de idosos tiveram mais domicílios alocados, de forma a produzir uma amostra mais representativa.

O número total de idosos alocados para o estudo considerou uma prevalência de 25\% para os sintomas depressivos $^{12}$, uma população estimada de 30.790 idosos $^{21}$, uma margem de erro de 5\% e um nível de confiança de 95\%. Considerando tratar-se de uma amostragem por conglomerados, o número identificado foi multiplicado por um fator de correção (deff) de 2 e acrescido de 15\% para eventuais perdas. Esses cálculos definiram uma amostra de, pelo menos, 614 idosos.

Os critérios de inclusão foram: ter idade igual ou superior a 60 anos e ser residente na área selecionada. Os critérios de exclusão foram: estar em uso de antidepressivos; incapacidade cognitiva, segundo a avaliação da família; déficit auditivo não corrigido que impedisse o entendimento das perguntas e recusa à participação na pesquisa por parte do idoso ou de seus familiares. A incapacidade cognitiva segundo a avaliação da família foi tomada como medida plausível diante da complexidade de se aplicar um instrumento específico para tal. Nesse caso, antes do início da entrevista, o instrumento de coleta de dados foi apresentado para os familiares, que julgaram se o idoso tinha condição de respondê-lo. Foram consideradas perdas os idosos não disponíveis em pelo menos três visitas, em dias e horários diferentes, mesmo com agendamento prévio.

A coleta de dados foi realizada no domicílio do idoso por entrevistadores de nível superior, previamente treinados e calibrados, que percorreram os setores censitários a partir de um ponto previamente definido, por sorteio, em cada setor censitário. Os instrumentos de coleta de dados utilizados foram baseados em estudos similares ${ }^{22}$ e foram previamente testados em estudo-piloto em um setor censitário especialmente sorteado e cujos dados não foram incluídos no trabalho final.

A variável-desfecho deste estudo - sintomas depressivos - foi obtida por meio da aplicação da Escala Geriátrica de Depressão com 15 questões (Geriatric Depression Scale GDS-15) ${ }^{23}$. Trata-se de instrumento já validado nacionalmente, composto por perguntas negativas/afirmativas, em que o resultado de 6 ou mais pontos identifica sintomatologia depressiva; dessa forma, o ponto de corte adotado foi $5 / 6$ (não caso/caso) 23,24.

As variáveis independentes estudadas foram: gênero, idade, cor da pele autorreferida, situação conjugal, arranjo familiar (condição de residir sozinho ou com outras pessoas), número de pessoas por domicílio, escolaridade, capacidade para leitura, renda própria, prática religiosa, tabagismo, ocorrência de queda no último ano, número de quedas, medo de cair, presença de morbidades crônicas autorreferidas [hipertensão, cardiopatias, acidente vascular encefálico, diabetes mellitus, artrites, osteoporose, asma, doença pulmonar obstrutiva crônica (DPOC)], presença de cuidador, autoavaliação da saúde, risco de quedas e fragilidade.

Para as variáveis demográficas e socioeconômicas, além daquelas relacionadas aos cuidados de saúde e morbidade referida, foram utilizadas questões do Vigitel/201122.

O risco de quedas foi obtido por meio do teste de mobilidade funcional Timed Get Up and $\mathrm{Go}^{25}$, realizado no domicílio dos idosos. Trata-se de um instrumento que avalia o equilíbrio sentado, transferências de sentado para a posição de pé, estabilidade na deambulação e mudança do curso da marcha sem utilizar estratégias compensatórias. A técnica consiste em solicitar ao idoso que se levante de uma cadeira (altura do assento de $45 \mathrm{~cm}$ e dos braços de $65 \mathrm{~cm}$ ), deambule 3 metros, retorne e sente-se novamente, enquanto se cronometra o tempo despendido na realização dessa tarefa. Em cada domicílio, os entrevistadores definiam o melhor espaço para aplicar o teste, considerando os requisitos necessários. Indivíduos independentes sem alterações no equilíbrio realizam o teste em 10 segundos ou menos; aqueles com independência em transferências básicas gastam 20 segundos ou menos. Já os indivíduos que necessitam de mais de 30 segundos para realizar o teste são dependentes em muitas atividades de vida diária e na mobilidade, apresentando riscos aumentados de cair. Foram considerados com menor risco de quedas os indivíduos que realizaram o teste em até 20 segundos e com alto risco de quedas aqueles que gastaram mais de 20 segundos ${ }^{25}$.

A fragilidade foi mensurada por meio da Edmonton Frail Scale $(E F S)^{26}$, um instrumento que avalia nove domínios cognição, estado de saúde, independência funcional, suporte social, uso de medicação, nutrição, humor, continência urinária e desempenho funcional -, distribuídos em 11 itens, com pontuação de 0 a 17. Para a análise estatística, os resultados da escala foram dicotomizados em dois níveis: sem fragilidade (escore final $\leq 6$ ) e com fragilidade (escore $>6)^{26}$.

As informações coletadas foram analisadas por meio do programa Statistical Package for the Social Sciences (SPSS), versão 17.0 (SPSS for Windows, Chicago, EUA). Inicialmente foi realizada análise descritiva dos dados. Em seguida, investi- 
gou-se a existência de associação estatística entre a variável dependente e demais variáveis. Para tanto, foram conduzidas análises bivariadas (teste qui-quadrado de Pearson), adotando-se nível de significância menor que 0,20 para inclusão das variáveis independentes no modelo múltiplo. O modelo final foi gerado por meio de análise de regressão logística múltipla e foram mantidas no modelo final as variáveis que apresentaram associação com sintomas depressivos até um nível de significância de 5\% ( $p<0,05)$.

O projeto desta pesquisa foi submetido ao Comitê de Ética em Pesquisa da Universidade Estadual de Montes Claros e aprovado mediante o parecer do processo de no 173.397. Todos os participantes foram orientados sobre a pesquisa e apresentaram sua anuência, com assinatura de Termo de Consentimento Livre e Esclarecido. Quando o idoso não podia assinar, fazia registro de sua digital ou alguém da família assinava o termo, consentindo a entrevista para coleta de dados.

\section{RESULTADOS}

Foram entrevistados 681 idosos, dos quais 42 foram excluídos por estarem em uso de antidepressivos. Ao final, foram avaliados 639 idosos, com idades entre 60 e 98 anos, sendo a média de 70,6 anos (DP $\pm 7,8$ ). A maior parte era do gênero feminino $(64,0 \%)$ e autodeclarados não brancos $(68,9 \%)$. Quase metade dos idosos era casada (49,5\%). A escolaridade média da amostra foi de 3,7 anos. As principais características sociodemográficas do grupo são apresentadas na tabela 1.

Dos idosos avaliados, a maior parte $(51,0 \%)$ descreveu sua saúde como razoável ou ruim e 89,5\% informaram ter passado por alguma consulta médica nos últimos 12 meses. O relato de quedas no último ano foi referido por $27,1 \%$ dos idosos e 12,8\% apresentaram alto risco de quedas mediante o teste de mobilidade funcional. Hipertensão foi a doença crônica mais presente na amostra estudada, sendo autorrelatada por 70,9\% dos idosos. As principais características do grupo em relação às condições de saúde e morbidades são apresentadas nas tabelas 2 e 3 , respectivamente.

A prevalência de sintomas depressivos foi de 27,5\% (IC 95\%: 24,1-31,1), sendo $29,1 \%$ no gênero feminino e $24,8 \%$ no masculino. Para idosos com 80 anos ou mais, a prevalência foi de $34,8 \%$, enquanto para os mais jovens, entre 60 e 79 anos, foi de $26,3 \%$.

Associações entre as variáveis independentes e sintomas depressivos são apresentadas nas tabelas 4 e 5 . Após análise de regressão múltipla, as variáveis que se mantiveram associadas a sintomas depressivos foram: não ter companheiro (situação conjugal) (OR=1,81; IC 95\% 1,214-2,713), não saber ler (OR = 1,84; IC 95\% 1,19-2,836), percepção negativa sobre a própria saúde (OR = 2,12; IC 95\% 1,373-3,256), tabagismo $(\mathrm{OR}=2,31$; IC 95\% 1,208-4,431), alto risco de quedas (OR =
1,78; IC 95\% 1,000-3,184) e fragilidade $(\mathrm{OR}=2,38$; IC 95\% $1,510-3,754)$

Tabela 1. Características sociodemográficas de idosos em Montes Claros (MG), 2013

\begin{tabular}{|c|c|c|}
\hline Variáveis & $n$ & $\%$ \\
\hline \multicolumn{3}{|l|}{ Gênero } \\
\hline Masculino & 230 & 36,0 \\
\hline Feminino & 409 & 64,0 \\
\hline \multicolumn{3}{|l|}{ Idade } \\
\hline 60 a 69 anos & 326 & 51,0 \\
\hline 70 a 79 anos & 221 & 34,6 \\
\hline 80 anos ou mais & 92 & 14,4 \\
\hline \multicolumn{3}{|l|}{ Cor da pele autorreferida } \\
\hline Parda & 366 & 57,3 \\
\hline Preta & 62 & 9,7 \\
\hline Branca & 199 & 31,1 \\
\hline Amarela & 12 & 1,9 \\
\hline \multicolumn{3}{|l|}{ Estado conjugal $(n=638)$} \\
\hline Solteiro & 42 & 6,6 \\
\hline Casado & 315 & 49,4 \\
\hline União estável & 16 & 2,5 \\
\hline Divorciado/separado & 49 & 7,7 \\
\hline Viúvo & 216 & 33,9 \\
\hline \multicolumn{3}{|l|}{ Prática religiosa } \\
\hline Sim & 528 & 82,6 \\
\hline Não & 111 & 17,4 \\
\hline \multicolumn{3}{|l|}{ Arranjo familiar $(n=638)$} \\
\hline Mora sozinho & 79 & 12,4 \\
\hline Mora somente com cônjuge & 112 & 17,5 \\
\hline Mora com outros familiares & 444 & 69,5 \\
\hline Mora com não familiares & 3 & 0,5 \\
\hline \multicolumn{3}{|l|}{ Renda própria } \\
\hline Sim & 570 & 89,2 \\
\hline Não & 69 & 10,8 \\
\hline \multicolumn{3}{|l|}{ Escolaridade } \\
\hline Nenhuma & 165 & 25,8 \\
\hline 1 a 4 anos & 319 & 49,9 \\
\hline 5 a 8 anos & 99 & 15,5 \\
\hline 9 a 11 anos & 24 & 3,8 \\
\hline 12 ou mais & 32 & 5,0 \\
\hline \multicolumn{3}{|c|}{ Capacidade para leitura (sabe ler) } \\
\hline Sim & 462 & 72,3 \\
\hline Não & 177 & 27,7 \\
\hline \multicolumn{3}{|c|}{ Número de pessoas por domicílio } \\
\hline Até 2 pessoas & 274 & 42,9 \\
\hline 3 a 6 pessoas & 349 & 54,6 \\
\hline 7 ou mais pessoas & 16 & 2,5 \\
\hline
\end{tabular}


Tabela 2. Condições de saúde e variáveis associadas entre idosos em Montes Claros (MG), 2013

\begin{tabular}{|c|c|c|}
\hline Variáveis & $\mathrm{n}$ & $\%$ \\
\hline \multicolumn{3}{|c|}{ Queda nos últimos 12 meses $(n=638)$} \\
\hline Sim & 173 & 27,1 \\
\hline Não & 465 & 72,9 \\
\hline \multicolumn{3}{|c|}{ Número de quedas nos últimos 12 meses $(n=173)$} \\
\hline Cinco ou mais & 22 & 3,4 \\
\hline Menos de cinco & 151 & 23,6 \\
\hline \multicolumn{3}{|l|}{ Medo de cair $(n=638)$} \\
\hline Sim & 451 & 70,6 \\
\hline Não & 187 & 29,3 \\
\hline \multicolumn{3}{|l|}{ Tabagismo } \\
\hline Sim & 56 & 8,8 \\
\hline Não & 583 & 91,2 \\
\hline \multicolumn{3}{|l|}{ Possui cuidador } \\
\hline Não & 537 & 84,0 \\
\hline Sim & 102 & 16,0 \\
\hline \multicolumn{3}{|l|}{ Fragilidade* } \\
\hline Frágil & 215 & 33,6 \\
\hline Não frágil & 424 & 66,4 \\
\hline \multicolumn{3}{|l|}{ Mobilidade funcional $^{* *}$} \\
\hline Alto risco de quedas & 82 & 12,8 \\
\hline Menor risco de quedas & 557 & 87,2 \\
\hline \multicolumn{3}{|l|}{ Autopercepção da saúde } \\
\hline Excelente/muito boa/boa & 313 & 49,0 \\
\hline Razoável & 264 & 41,3 \\
\hline Ruim & 62 & 9,7 \\
\hline
\end{tabular}

*Edmonton Frail Scale; ** Timed get out and go test.

\section{DISCUSSÃO}

A literatura revela que a prevalência de sintomas depressivos em idosos comunitários pode apresentar grande variabilidade tanto entre países quanto entre diferentes comunidades dentro de um mesmo país ${ }^{13}$. Achados nacionais variam de 13\%, conforme estudo realizado em São Paulo com 1.563 idosos $^{11}$, a 38,5\%, segundo pesquisa com 1.510 idosos, na cidade de Bambuí, MG ${ }^{8}$. É possível que parte dessa variabilidade seja atribuída à metodologia utilizada e à forma de mensuração, isto é, ao instrumento utilizado.

Neste estudo, a prevalência de sintomas depressivos se mostrou ligeiramente superior àquela observada por Maciel e Guerra, em pesquisa realizada no nordeste brasileiro' ${ }^{12}$, com amostra de 310 idosos, utilizando também a GDS-15. A prevalência observada neste trabalho também é um pouco maior do que a registrada em outro estudo de base populacional no sul do Brasil, com amostra de 1.656 idosos, referenciando o mesmo instrumento de coleta de dados ${ }^{18}$.
Tabela 3. Morbidades autorreferidas por idosos em Montes Claros (MG), 2013

\begin{tabular}{|c|c|c|}
\hline Variáveis & n & $\%$ \\
\hline \multicolumn{3}{|c|}{ Hipertensão ( $\mathrm{n}=638$ ) } \\
\hline Sim & 453 & 70,9 \\
\hline Não & 185 & 29,0 \\
\hline \multicolumn{3}{|c|}{ Diabetes $(n=637)$} \\
\hline Sim & 143 & 22,4 \\
\hline Não & 494 & 77,6 \\
\hline \multicolumn{3}{|c|}{ Cardiopatias $(n=627)$} \\
\hline Sim & 141 & 22,5 \\
\hline Não & 486 & 77,5 \\
\hline \multicolumn{3}{|c|}{ Artrites/reumatismo $(n=627)$} \\
\hline Sim & 220 & 35,1 \\
\hline Não & 407 & 64,9 \\
\hline \multicolumn{3}{|c|}{ Osteoporose $(n=619)$} \\
\hline Sim & 154 & 24,9 \\
\hline Não & 465 & 75,1 \\
\hline \multicolumn{3}{|c|}{ Acidente vascular encefálico ( $n=632$ ) } \\
\hline Sim & 33 & 5,2 \\
\hline Não & 599 & 94,8 \\
\hline \multicolumn{3}{|c|}{ Doença pulmonar obstrutiva crônica $(\mathrm{n}=638)$} \\
\hline Sim & 27 & 4,2 \\
\hline Não & 611 & 95,8 \\
\hline \multicolumn{3}{|c|}{ Asma $(n=638)$} \\
\hline Sim & 30 & 4,7 \\
\hline Não & 608 & 95,3 \\
\hline
\end{tabular}

Alvarenga et al., em investigação epidemiológica realizada com 503 idosos na região Centro-Oeste do Brasil, com uso da GDS-15, registraram prevalência de sintomas depressivos de $34,4 \%{ }^{27}$. Todavia, é preciso salientar que o estudo foi restrito a idosos cadastrados em equipes da Estratégia Saúde da Família.

A utilização de outros instrumentos registra resultados mais distantes do que se observou no presente trabalho. $\mathrm{O}$ estudo de Bambuí, em Minas Gerais, foi realizado utilizando o General Health Questionnaire, apontando prevalência de sintomas depressivos de 38,5\%, enquanto outro estudo conduzido em São Paulo ${ }^{16}$, com uso do Short Psychiatric Evaluation Schedule, mostrou prevalência de 21,1\%. A utilização do questionário Brazil Old Age Schedule, em outro estudo de base populacional no sul do Brasi ${ }^{28}$, registrou prevalência de $19,7 \%$.

Estudos internacionais também apresentam grande variabilidade, em decorrência dos instrumentos e da população avaliada. Richardson et al., em estudo realizado em Nova 
Tabela 4. Análise bivariada dos sintomas depressivos em idosos, segundo as variáveis sociodemográficas

\begin{tabular}{|c|c|c|c|c|c|c|}
\hline \multirow{3}{*}{ Variáveis } & \multicolumn{4}{|c|}{ GDS } & \multirow{3}{*}{ OR (IC95\%) } & \multirow{3}{*}{ Valor $P$} \\
\hline & \multicolumn{2}{|c|}{$\geq 6$} & \multicolumn{2}{|c|}{$<5$} & & \\
\hline & (n) & $(\%)$ & (n) & $(\%)$ & & \\
\hline Gênero & & & & & & 0,241 \\
\hline Feminino & 119 & 29,1 & 290 & 70,9 & $1,24(0,86-1,80)$ & \\
\hline Masculino & 57 & 24,8 & 177 & 74,1 & 1 & \\
\hline Idade & & & & & & 0,093 \\
\hline Maior de 80 anos & 32 & 34,8 & 60 & 65,2 & $1,50(0,93-2,387)$ & \\
\hline De 60 a 80 anos & 144 & 26,3 & 403 & 73,7 & 1 & \\
\hline Cor da pele autorreferida & & & & & & 0,877 \\
\hline Não branco & 122 & 27,7 & 318 & 72,3 & $1,03(0,71-1,50)$ & \\
\hline Branco & 54 & 27,1 & 145 & 72,9 & 1 & \\
\hline Situação conjugal & & & & & & $<0,001$ \\
\hline Sem companheiro & 105 & 34,1 & 203 & 65,9 & $1,89(1,33-2,69)$ & \\
\hline Com companheiro & 71 & 21,5 & 260 & 78,5 & 1 & \\
\hline Prática religiosa & & & & & & 0,028 \\
\hline Não & 40 & 36,0 & 71 & 64,0 & $1,62(1,05-2,51)$ & \\
\hline Sim & 136 & 25,8 & 392 & 74,2 & 1 & \\
\hline Arranjo familiar & & & & & & 0,006 \\
\hline Reside sozinho & 32 & 40,5 & 47 & 59,5 & $1,96(1,20-3,19)$ & \\
\hline Não reside sozinho & 144 & 25,8 & 415 & 74,2 & 1 & \\
\hline Renda própria & & & & & & 0,762 \\
\hline Não & 19 & 27,5 & 50 & 72,5 & $1,00(0,57-1,75)$ & \\
\hline Sim & 157 & 27,5 & 413 & 72,5 & 1 & \\
\hline Escolaridade & & & & & & 0,112 \\
\hline Quatro anos ou menos & 141 & 29,1 & 343 & 70,9 & $1,41(0,92-2,15)$ & \\
\hline Mais de 4 anos & 35 & 22,6 & 120 & 77,4 & 1 & \\
\hline Sabe ler & & & & & & $<0,001$ \\
\hline Não & 69 & 39,0 & 108 & 61,0 & $2,12(1,46-3,07)$ & \\
\hline Sim & 107 & 23,2 & 355 & 76,8 & 1 & \\
\hline Número de pessoas por d & & & & & & 0,740 \\
\hline Quatro ou mais pessoas & 61 & 26,8 & 167 & 73,2 & $0,94(0,65-1,35)$ & \\
\hline Menos de 4 pessoas & 115 & 28,0 & 296 & 72,0 & 1 & \\
\hline
\end{tabular}

lorque, com 378 idosos, apontaram que quase um terço dos indivíduos estudados (31,0\%) apresentou sintomas depressivos por meio do Patient Health Questionnaire-93.

Utilizando a GDS-15, um estudo japonês ${ }^{14}$ com cerca de 5.400 idosos comunitários, de 65 anos ou mais e residentes em áreas rurais, registrou uma prevalência de sintomas depressivos clinicamente significativos de 33,5\%. Já um estudo alemão ${ }^{15}$ com cerca de 3.300 idosos de 75 anos ou mais, utilizando a mesma escala e pontos de corte, registrou uma prevalência de 9,7\%.

As diversas prevalências identificadas pelos estudos, independentemente de apresentarem variabilidade atribuída aos tamanhos amostrais, às diferentes escalas e pontos de corte utilizados para sintomas depressivos, aos pontos de corte de idade como critério de inclusão ou às peculiaridades regionais de cada grupo populacional estudado, salientam a relevância do tema. As prevalências registradas não são desprezíveis e alertam para a necessidade de cuidados especiais com a população idosa, sobretudo considerando o acelerado processo de envelhecimento populacional que o Brasil vivencia nos últimos anos ${ }^{29}$.

A investigação dos fatores associados aos sintomas depressivos revelou como fatores de risco: aspectos relacionados às condições de saúde (percepção negativa sobre a própria saúde, alto risco de quedas e fragilidade), aspectos sociais (não ter companheiro, não saber ler) e hábito de vida (tabagismo). 
Tabela 5. Análise bivariada dos sintomas depressivos em idosos, segundo condições de saúde e morbidades

\begin{tabular}{|c|c|c|c|c|}
\hline Variáveis & $\begin{array}{l}\text { Sintomas depressivos presentes } \\
\mathrm{n}(\%)\end{array}$ & $\begin{array}{l}\text { Sintomas depressivos ausentes } \\
\mathrm{n}(\%)\end{array}$ & OR (IC 95\%) & Valor $P$ \\
\hline \multicolumn{5}{|c|}{ Queda nos últimos 12 meses } \\
\hline Sim & $58(33,7 \%)$ & $114(66,3 \%)$ & $1,496(1,024-2,185)$ & 0,037 \\
\hline Não & $118(25,4 \%)$ & $347(74,6 \%)$ & 1 & \\
\hline \multicolumn{5}{|c|}{ Número de quedas nos últimos 12 meses } \\
\hline Cinco ou mais & $11(50 \%)$ & $11(50 \%)$ & $2,020(0,820-4,978)$ & 0,121 \\
\hline Menos de cinco & $50(33,1 \%)$ & $112(66,1 \%)$ & 1 & \\
\hline \multicolumn{5}{|l|}{ Medo de cair } \\
\hline Sim & $138(30,6 \%)$ & $313(69,4 \%)$ & $1,729(1,149-2,601)$ & 0,008 \\
\hline Não & $38(20,3 \%)$ & $149(79,4 \%)$ & 1 & \\
\hline \multicolumn{5}{|l|}{ Hipertensão } \\
\hline Sim & $129(28,5 \%)$ & $324(70,2 \%)$ & $1,169(0,792-1,725)$ & 0,431 \\
\hline Não & $47(25,4 \%)$ & $138(74,6 \%)$ & 1 & \\
\hline \multicolumn{5}{|l|}{ Diabetes } \\
\hline Sim & $49(34,3 \%)$ & $94(65,7 \%)$ & $1,522(1,020-2,272)$ & 0,039 \\
\hline Não & $126(25,5 \%)$ & $368(74,5 \%)$ & 1 & \\
\hline \multicolumn{5}{|l|}{ Cardiopatias } \\
\hline Sim & $39(27,7 \%)$ & $102(72,3 \%)$ & $1,047(0,688-1,594)$ & 0,830 \\
\hline Não & $130(26,7 \%)$ & $356(73,3 \%)$ & 1 & \\
\hline \multicolumn{5}{|l|}{ Artrites/reumatismo } \\
\hline Sim & $67(30,5 \%)$ & $153(69,5 \%)$ & $1,309(0,910-1,885)$ & 0,146 \\
\hline Não & $102(25,1 \%)$ & $305(74,9 \%)$ & 1 & \\
\hline \multicolumn{5}{|l|}{ Osteoporose } \\
\hline Sim & $51(33,1 \%)$ & $103(66,9 \%)$ & $1,525(1,025-2,267)$ & 0,036 \\
\hline Não & $114(24,4 \%)$ & $351(75,5 \%)$ & 1 & \\
\hline \multicolumn{5}{|c|}{ Acidente vascular encefálico } \\
\hline Sim & $13(39,4 \%)$ & $20(60,6 \%)$ & $1,783(0,867-3,669)$ & 0,112 \\
\hline Não & $160(26,7 \%)$ & $439(73,3 \%)$ & 1 & \\
\hline \multicolumn{5}{|l|}{ Asma } \\
\hline Sim & $7(23,3 \%)$ & $23(76,7 \%)$ & $0,791(0,333-1,877)$ & 0,593 \\
\hline Não & $169(27,8 \%)$ & $439(72,2 \%)$ & 1 & \\
\hline \multicolumn{5}{|l|}{ Tabagismo } \\
\hline Sim & $27(48,2 \%)$ & $29(51,8 \%)$ & $2,712(1,555-4,730)$ & $<0,001$ \\
\hline Não & $149(25,6 \%)$ & $434(74,4 \%)$ & 1 & \\
\hline \multicolumn{5}{|l|}{ Possui cuidador } \\
\hline Sim & $43(42,2 \%)$ & $49(57,8 \%)$ & $2,214(1,427-3,434)$ & $<0,001$ \\
\hline Não & $133(24,8 \%)$ & $404(75,2 \%)$ & 1 & \\
\hline \multicolumn{5}{|l|}{ Fragilidade } \\
\hline Frágil & $102(47,4 \%)$ & $113(52,6 \%)$ & $4,269(2,958-6,060)$ & $<0,001$ \\
\hline Não frágil & $74(17,5 \%)$ & $350(82,5 \%)$ & 1 & \\
\hline \multicolumn{5}{|l|}{ Mobilidade funcional } \\
\hline Alto risco de quedas & $42(51,2 \%)$ & $45(48,8 \%)$ & $3,315(2,062-5,328)$ & $<0,001$ \\
\hline Menor risco de quedas & $134(24,1 \%)$ & $423(75,9 \%)$ & 1 & \\
\hline \multicolumn{5}{|l|}{ Autopercepção da saúde } \\
\hline Razoável/ruim & $120(36,8 \%)$ & $206(63,2 \%)$ & $2,673(1,853-3,856)$ & $<0,001$ \\
\hline Boa/excelente & $56(17,9 \%)$ & $257(82,1 \%)$ & 1 & \\
\hline
\end{tabular}


A percepção negativa sobre a própria saúde, assim como em outros trabalhos ${ }^{8,12,17,18}$, mostrou-se associada à sintomatologia depressiva. A percepção da saúde é um dado subjetivo e diz respeito à forma como o indivíduo vê seu estado geral, o que inclui as dimensões biológica, psicológica e social ${ }^{12}$. Fatores associados ao surgimento de sintomas depressivos podem concomitantemente afetar a autopercepção de saúde do indivíduo, o que justificaria a associação. Em estudo realizado em Florianópolis, o autor defende que o próprio relato ruim sobre a saúde pode ser entendido como sintoma depressivo ${ }^{18}$.

A relação entre os sintomas depressivos e a restrição da mobilidade por medo de cair, assim como as quedas recorrentes, está bem relatada na literatura ${ }^{30,31}$. No presente trabalho, o alto risco de quedas, estimado pelo teste de mobilidade funcional, se mostrou estatisticamente associado aos sintomas depressivos. Entretanto, para o relato de quedas nos últimos 12 meses, a associação não se manteve significante. É possível que o relato de quedas tenha sido subestimado em razão da limitação de memória. Todavia, esse aspecto não foi avaliado no presente estudo.

A sintomatologia depressiva associou-se com fragilidade, de forma semelhante a outros estudos ${ }^{32-34}$. Essa associação poderia estar vinculada à sobreposição de características coexistentes em ambas as condições de saúde, por exemplo, a inatividade, a perda de peso, a exaustão e o nível reduzido de atividade física ${ }^{33,34}$.

No que diz respeito à situação conjugal, a condição de não ter companheiro (solteiro/viúvo/divorciado) mostrou-se associada com a presença de sintomas depressivos. Estudo realizado na cidade de Bambuí identificou associação semeIhante para a condição de solteiro e separado ${ }^{8}$.

A associação de sintomatologia depressiva com analfabetismo, já encontrada em outro trabalho ${ }^{12}$, foi corroborada neste estudo. Acredita-se que o nível educacional possibilita que o indivíduo amplie os recursos de enfrentamento das situações estressantes da vida ${ }^{8,10}$, dessa forma indivíduos analfabetos apresentariam maior risco de desenvolver sintomas depressivos.

O tabagismo mostrou-se associado à presença de sintomas depressivos, resultado concordante com outros estu$\operatorname{dos}^{15,19}$. Sabe-se que a nicotina interfere no funcionamento neuroendócrino e, dessa forma, pode influenciar o quadro psicopatológico. Provavelmente, as pessoas que relatam ansiedade e depressão sentem-se bem fumando, porque a nicotina é ansiolítica e contribui para o alívio dos sintomas ${ }^{35}$.

Por outro lado, estudos demonstram que a doença cerebrovascular pode predispor, precipitar ou perpetuar a síndrome depressiva nos idosos, mediante a interrupção dos circuitos frontoestriatais, que ligam o córtex pré-frontal aos gânglios basais ${ }^{36}$. Nessa perspectiva, o tabagismo poderia estar associado a sintomas depressivos, por causa de seu potencial aterogênico na patogênese dos eventos cerebrovasculares. Contudo, segundo Gazalle et al., o papel do taba- gismo na ocorrência da depressão é controverso e estudos epidemiológicos e neurobiológicos focados exclusivamente na relação tabaco versus depressão são necessários para elucidar essa associação ${ }^{19}$.

Diversos estudos têm demonstrado relação entre sintomas depressivos e idade em idosos ${ }^{8,9,12}$; neste trabalho, essa associação não foi estatisticamente significativa. Segundo Castro-Costa et al., a associação entre envelhecimento e depressão tem sido inconsistente e tende a desaparecer após o ajuste para a incapacidade funcional, distúrbios médicos e comorbidades ${ }^{8}$.

A associação entre gênero feminino e sintomas depressivos em idosos foi um achado comum na literatura ${ }^{8-11}$. Por outro lado, investigações epidemiológicas de grande tamanho amostral, como um estudo realizado no sul do Brasil com 7.040 idosos $^{17}$ e outro conduzido na Alemanha com 3.327 idosos $^{15}$, não demonstraram essa associação. $O$ presente trabalho também não constatou influência do gênero na prevalência de sintomas depressivos em idosos. Mais estudos ainda são necessários para determinar se há uma real associação entre sintomas depressivos e gênero na terceira idade.

Os resultados deste estudo devem ser considerados à luz de algumas limitações. Trata-se de pesquisa conduzida em um único município, no qual a identificação das morbidades foi realizada por meio do autorrelato, fato que pode ter propiciado uma subestimativa da real prevalência das condições de morbidade. Outro aspecto diz respeito ao local de coleta de casos - o próprio domicílio do idoso -, o que pode ter gerado algum viés, pois idosos mais ativos estariam trabaIhando e não disponíveis para responder ao inquérito, mesmo depois de repetidas visitas. Todavia, é preciso considerar que o estudo foi conduzido com uma amostra relativamente grande, alocada em 42 setores censitários, incorporando diferentes estratos sociais da população e que, para a coleta de dados, foram utilizados instrumentos padronizados.

A identificação de fatores associados aos sintomas depressivos em idosos, quando usada para reconhecer populações em alto risco, pode se constituir no passo fundamental para o planejamento de intervenções de base populacional que visem reduzir a prevalência de sintomas depressivos e suas complicações.

O uso rotineiro, por parte dos profissionais de saúde, de instrumentos de triagem de depressão, facilmente aplicáveis na prática clínica, permitiria não só o diagnóstico de muitos casos da doença, que passam despercebidos e influenciam adversamente a qualidade de vida dos pacientes, como também o prognóstico das comorbidades existentes, as quais contribuem para maior mortalidade, seja pelo aumento do risco de suicídio, seja pela evolução desfavorável das doenças crônicas existentes ${ }^{20}$. Novos estudos são desejáveis para essa área do conhecimento, buscando novas associações que permitam intervenções precoces e mais efetivas sobre a saúde mental dos idosos. 


\section{CONCLUSÕES}

A prevalência de sintomas depressivos identificada neste estudo foi alta e alerta para a necessidade de maiores cuidados com a população idosa. Especial atenção deve ser dada àqueles idosos que apresentam percepção negativa da saúde, tabagismo, alto risco de quedas, fragilidade, assim como aos que não sabem ler e não têm companheiro(a), por serem condições associadas à presença de sintomatologia depressiva.

\section{CONTRIBUIÇÕES INDIVIDUAIS}

Gizele Carmem Fagundes Ramos - Contribuiu significativamente na concepção e desenho do estudo, na análise e interpretação dos dados e na elaboração e revisão crítica do artigo, e aprovou a versão final a ser publicada.

Jair Almeida Carneiro - Contribuiu significativamente na concepção e desenho do estudo, na análise e interpretação dos dados e na elaboração do artigo, e aprovou sua versão final a ser publicada.

Ana Teresa Fernandes Barbosa - Contribuiu significativamente na análise e interpretação dos dados, na elaboração do artigo e na revisão crítica do seu conteúdo intelectual, e aprovou sua versão final a ser publicada.

José Marcio Girardi Mendonça - Contribuiu na concepção e desenho do estudo e na elaboração do artigo, e aprovou a versão final a ser publicada.

Antônio Prates Caldeira - Contribuiu significativamente na concepção e desenho do estudo, na análise e interpretação dos dados e na elaboração e revisão crítica do artigo, e aprovou a versão final a ser publicada.

\section{CONFLITOS DE INTERESSE}

Nenhum dos autores possui conflitos de interesse a serem declarados.

\section{AGRADECIMENTOS}

À Fundação de Amparo à Pesquisa do Estado de Minas Gerais (Fapemig), pelo suporte financeiro assegurado para a realização desta pesquisa.

\section{REFERÊNCIAS}

1. Moraes EN. Princípios básicos de geriatria e gerontologia. Belo Horizonte: Coopmed; 2008.

2. Buchanan D, Tourigny-Rivard MF, Cappelliez P, Frank C, Janikowski P, Spanjevic L, et al. National guidelines for seniors' mental health: the assessment and treatment of depression. Can J Geriatr. 2006;9(2):S52-8.
3. Richardson TM, Friedman B, Podgorski C, Knox K, Fisher S, He H, et al. Depression and its correlates among older adults accessing aging services. J Geriatr Psychiatry. 2012;20(4):346-54.

4. Ferrari JF, Dalacorte RR. Uso da Escala de Depressão Geriátrica de Yesavage para avaliar a prevalência de depressão em idosos hospitalizados. Sci Med. 2007;17(1):3-8.

5. Malloy-Diniz LF, Fuentes D, Consenza RM. Neuropsicologia do envelhecimento: uma abordagem multidimensional. Porto Alegre: Artmed; 2013.

6. Barcelos-Ferreira R, lzbicki R, Steffens DC, Bottino CM. Depressive morbidity and gender in community-dwelling Brazilian elderly: systematic review and meta-analysis. Int Psychogeriatr. 2010;22(5):712-26.

7. Harman JS, Veazie PJ, Lynsess JM. Primary care physician office visits for depression by older Americans. J Gen Intern Med. 2006;21(9):926-30.

8. Castro-Costa E, Lima-Costa MF, Carvalhais S, Firmo JOA, Uchoa E. Factors associated with depressive symptoms measured by the 12-item General Health Questionnaire in Community-Dwelling Older Adults (The Bambuí Health Aging Study). Rev Bras Psiquiatr. 2008;30(2):104-9.

9. Barcelos-Ferreira R, Izbicki R, Steffens DC, Bottino CM. Depressive morbidity and gender in community-dwelling Brazilian elderly: systematic review and meta-analysis. Int Psychogeriatr. 2010:22(5):712-26.

10. Batistoni SST, Neri AL, Cupertino APFB. Medidas prospectivas de sintomas depressivos entre idosos residentes na comunidade. Rev Saúde Pública. 2010;44(6):1137-43.

11. Barcelos-Ferreira R, Pinto JA Jr, Nakano EY, Steffens DC, LitvoC J, Bottino CM. Clinically significant depressive symptoms and associated factors in community elderly subjects from Sao Paulo, Brazil. Am J Geriatr Psychiatry. 2009;17(7):582-90.

12. Maciel ACC, Guerra RO. Prevalência e fatores associados à sintomatologia depressiva em idosos residentes no Nordeste do Brasil. J Bras Psiquiatr. 2006;55(1):26-33.

13. Hoffmann EJ, Ribeiro F, Farnese JM, Lima EWB. Sintomas depressivos e fatores associados entre idosos residentes em uma comunidade no norte de Minas Gerais, Brasil. J Bras Psiquiatr. 2010:59(3):190-7.

14. Wada T, Ishine M, Sakagami T, Okumiya K, Fujisawa M, Murakami S, et al. Depression in Japanese community-dwelling elderly - prevalence and association with ADL and QOL. Arch Gerontol Geriatr. 2004;39(1):15-23.

15. Weyerer S, Eifflaender-Gorfer S, Köhler L, Jessen F, Maier W, Fuchs A, et al. Prevalence and risk factors for depression in non-demented primary care attenders aged 75 years and older. J Affect Disord. 2008;111(2-3):153-63.

16. Lima MTR, Silva RS, Ramos LR. Fatores associados à sintomatologia depressiva numa coorte urbana de idosos. J Bras Psiquiatr. 2009:58(1):1-7.

17. Blay SL, Andreoli SB, Fillenbaum GG, Gastal FL. Depression morbidity in later life: prevalence and correlates in a developing country. Am J Geriatr Psychiatry. 2007;15(9):790-9.

18. Borges LJ, Benedetti TRB, Xavier AJ, d'Orsi E. Fatores associados aos sintomas depressivos em idosos: estudo Epi Floripa. Rev Saúde Pública. 2013;47(4):701-10.

19. Gazalle FK, Lima MS, Tavares BF, Hallal PC. Sintomas depressivos e fatores associados em população idosa no sul do Brasil. Rev Saúde Pública. 2004;38(3):365-71.

20. Duarte MB, Rego MAV. Comorbidade entre depressão e doenças clínicas em um ambulatório de geriatria. Cad Saude Publica. 2007;23(3):691-700.

21. Instituto Brasileiro de Geografia e Estatística (IBGE). Sinopse do Senso Demográfico de 2010. Rio de Janeiro; 2011.

22. Brasil. Ministério da Saúde. Secretaria de Vigilância em Saúde. Vigitel Brasil 2011: Vigilância de Fatores de Risco e Proteção para Doenças Crônicas por Inquérito Telefônico. Brasília: Ministério da Saúde; 2012.

23. Almeida OP, Almeida SA. Confiabilidade da versão brasileira da Escala de Depressão em Geriatria (GDS) versão reduzida. Arq Neuro-Psiquiatr. 1999;57(2-B):421-6.

24. Ferrari JF, Dalacorte RR. Uso da Escala de Depressão Geriátrica de Yesavage para avaliar a prevalência de depressão em idosos hospitalizados. Sci Med. 2007;17(1):3-8.

25. Oliveira DLC, Goretti LC, Pereira LSM. 0 desempenho de idosos institucionalizados com alterações cognitivas em atividades de vida diária e mobilidade: estudo piloto. Rev Bras Fisioter. 2006;10(1):91-6.

26. Rolfson DB, Majumdar SR, Tsuyuki RT, Tahir A, Rockwood K. Validity and reliability of the Edmonton Frail Scale. Age Ageing. 2006;35(5):526-9. 
27. Alvarenga MRM, Oliveira MAC, Faccenda 0. Sintomas depressivos em idosos: análise dos itens da Escala de Depressão Geriátrica. Acta Paul Enferm. 2012;25(4):497-503.

28. Benedetti TRB, Borges LJ, Petroski EL, Gonçalves LHT. Atividade física e estado de saúde mental de idosos. Rev Saúde Pública. 2008;42(2):302-7.

29. Brasil. Ministério da Saúde (MS). Informe de situação e tendências: demografia e saúde. Rede Interagencial de Informações para Saúde. Braślia: MS; 2009.

30. Ricci NA, Gonçalves DFF, Coimbra IB, Coimbra AMV. Fatores associados ao histórico de quedas de idosos assistidos pelo programa de saúde da família. Saúde Soc São Paulo. 2010;19(4):898-909.

31. Dias RC, Freire MTF, Santos EG, Vieira RA, Dias JMD, Perracini MR. Characteristics associated with activity restriction induced by fear of falling in community-dwelling elderly. Rev Bras Fisioter. 2011;15(5):406-13.
32. Drey $M$, Wehr $H$, Wehr $G$, Uter W, Lang F, Rupprecht $R$, et al. The frailty syndrome in general practitioner care: a pilot study. Z Gerontol Geriatr. 2011;44(1):48-54.

33. Woods NF, LaCroix AZ, Gray SL, Aragaki A, Cochrane BB, Brunner RL, et al. Frailty: emergence and consequences in women aged 65 and older in the Women's Health Initiative Observational Study. J Am Geriatr Soc. 2005;53(8):1321-30.

34. Vieira RA, Guerra RO, Giacomin KC, Vasconcelos KSS, Andrade ACS, Pereira LSM, et. al. Prevalência de fragilidade e fatores associados em idosos comunitários de Belo Horizonte, Minas Gerais, Brasil: dados do Estudo FIBRA. Cad Saude Publica. 2013;29(8):1631-43.

35. Zaitune MPA, Barros MBA, Lima MGF, César CLG, Carandina L, Goldbaum M, et al. Fatores associados ao tabagismo em idosos: inquérito de Saúde no Estado de São Paulo (ISA-SP). Cad Saude Publica. 2012;28(3):583-95.

36. Fernandes APS. Lesão da substância branca cerebral (leucoaraiose) [dissertação]. Covilhã: Universidade da Beira Interior; 2012 